\title{
Comparative occlusal study analog and digital - a pilot study
}

\author{
Smaranda Buduru' ${ }^{1,2}$, Manuela Taut ${ }^{2}$, Cecilia Bacali', Manuela Manziuc' ${ }^{1}$, Tudor Serban ${ }^{3}$, \\ Cristian Culcitchi², Mihai Mitariü, Marius Negucioiu' \\ ${ }^{1}$ Department of Prosthodontics, "Iuliu Hatieganu" University of Medicine and Pharmacy, Cluj-Napoca, Romania \\ ${ }^{2}$ Stomestet Clinic, Cluj-Napoca, Romania \\ ${ }^{3}$ Faculty of Dentistry, "Iuliu Hatieganu" University of Medicine and Pharmacy, Cluj-Napoca, Romania \\ 4 "Lucian Blaga" University of Medicine and Pharmacy, Sibiu, Romania
}

\begin{abstract}
The aim of this study was to determine whether or not the analysis of maximal intercuspation, protrusive and laterotrusive movements requires an additional digital method of occlusal diagnosis using the T-Scan ${ }^{\mathrm{TM}} \mathrm{Novus}^{\mathrm{TM}}$ device (TekScan). In this observational study, we retrospectively examined a number of 10 subjects which were divided into 2 groups: the 1st group involved patients with intact dental arches and the 2 nd group with patients that had received implant-supported restorations. The methods of analysis involved the calibrated articulating paper of 200 and 40 micrometers, and also the T-Scan ${ }^{\mathrm{TM}}$ Novus $^{\mathrm{TM}}$ device (TekScan). The following data were collected: contact points in maximum intercuspation (functional and premature contacts), the pairs of teeth performing protrusion and right/left laterotrusion, active/passive interferences and premature contacts during protrusion and right/left laterotrusion. Descriptive statistics were obtained using the MedCalc Statistical Software version 19.2.6 (MedCalc Software bv, Ostend, Belgium) and Microsoft Excel for MAC 2011. It was tested if a statistically significant difference existed between the diagnostic methods regarding the number of contact points. The $T$ test for independent samples without the assumption of equal variances and the Mann-Whitney test was used (to which we reported the medians of the 2 subgroups and the $P$ value adjusted for equalities) and also the magnitude of association using the Chi-square test ( $p$ value) was measured. Also it was studied the correlation between the number of premature contacts and the number of contact points, respectively, obtained by each diagnostic method, and we expressed the results through the Spearman correlation coefficient. The analysis of the maximum intercuspation, of the protrusive and the laterotrusive movements has shown comparable results using the two examination methods. There were no statistically significant differences between the two methods of analyzing dental occlusion.
\end{abstract}

Keywords: occlusion analysis, T-Scan, articulating paper

\section{INTRODUCTION}

Although we are witnessing a paradigm shift in dentistry that is aiming towards digitization in both diagnostic and treatment stages, the analysis of dental occlusion predominantly uses the traditional diagnostic method (1-3). Although the importance of occlusal diagnosis is especially significant in prosthodontics, implantology and orthodontics, the articulating paper (AP) of various thicknesses remains the most commonly used method (4-6).
The new methods of diagnosing dental occlusion have become available in the last decade, among which the T-Scan Novus v10 computerized occlusal analysis system (Tekscan Inc., South Boston, MA USA) managed to record the distribution of occlusal contacts and sequential occlusal forces (7-9). Additionally, the T-Scan system records the time of occlusion and disocclusion in various mandibular reference movements (10).

The T-Scan HD sensor can acquire 256 levels of occlusal forces at the contact points as the patient 
bites down. The dedicated software processes and displays the occlusal forces in real time in 2D and 3D format, to be used in the diagnosis of excessive occlusal forces. The sensor is 100-micrometers thick $(11,12)$.

Accordingly, a pilot study was conducted to evaluate whether or not the analysis of dental occlusion requires an additional digital method of occlusal diagnosis using the T-Scan ${ }^{\mathrm{TM}}$ Novus $^{\mathrm{TM}}$ device (TekScan). For this purpose, the analysis of maximal intercuspation, protrusive and laterotrusive movements was performed using calibrated articulating paper and T-Scan Novus v10 respectively, then these two diagnostic methods were compared.

\section{MATERIALS AND METHODS}

In this observational, retrospective study, we examined 10 subjects (one man and nine women) aged between 25 and 50 years. The subjects were divided into 2 groups: 1st group with subjects having intact dental arches and the 2nd group with subjects having implant-supported restorations, such as single crowns or total bridges. The study received the approval of the Ethical Comittee of the "Iuliu Hatieganu" University of Medicine and Pharmacy Cluj-Napoca no 52/09.03.2021.

The inclusion criteria for group 1 were: the presence of at least 10 teeth/arch, the presence of dental restorations such as crowns, inlays, composite fillings, and the presence of functional relationships of static occlusion at the level of the incisors and canines.

The exclusion criteria for group 1 were presence of non-functional relationships of static occlusion at the incisors: sagittal and vertical open bite, deep bite, crossbite, edge-to-edge occlusion, and presence of non-functional relationships of static occlusion in canines: transversal and vertical open bite, crossbite.

The inclusion criteria for group 2 were: the presence of Kennedy classes I, II, III and IV edentulous ridges, that had been prosthetically restored using implant-supported crowns and/or bridges, the presence of total edentation that had also been restored using fixed implant-supported complete prosthetics, the presence of functional relations of static occlusion in incisors and canines.

The exclusion criteria for group 2 were: the presence of non-restored edentations, the presence of non-functional relationships of static occlusion in incisors and canines.

As evaluation methods, we included two objective methods for the analysis of dental occlusion: the calibrated articulating paper of 200 and $40 \mathrm{mi}-$ crometers and the T-Scan Novus v10 device, respectively. We evaluated whether or not the analog analysis (using articulating paper) of maximal intercuspation, protrusive and laterotrusive movements requires an additional digital method of occlusal diagnosis using the T-Scan Novus v10 device.

In the maximum intercuspation analysis, we recorded the contact points between the maxillary and mandibular supporting cusps and the premature contacts in maximum intercuspation in both groups of subjects using both the analogue method with the $40-\mu \mathrm{m}$ calibrated articulating paper and the digital method with the T-Scan Novus device. The premature contact points with the $40-\mu \mathrm{m}$ articulating paper were those left by the articulating paper on the occlusal surface with a higher color intensity or those in the form of a colored circle with the colorless center. Premature contacts at maximum intercuspation using T-Scan Novus were recorded in the pink-red color spectrum.

The pairs of teeth that performed the protrusion, right/left laterotrusion, and the ones that displayed active/passive interferences, active/passive premature contacts in protrusion and right/left laterotrusion were recorded for both groups using the analogue method with calibrated $40-\mu \mathrm{m}$ articulating paper and the digital method using the T-Scan Novus v10 device.

Participants' data were centralized in an Excel spreadsheet. Descriptive statistics were used to evaluate the characteristics of the subjects. The statistical analysis was performed using MedCalc Statistical Software version 19.2.6 (MedCalc Software bv, Ostend, Belgium; https://www.medcalc.org; 2020) and Microsoft Excel for MAC 2011.

We studied the distribution of contact points and premature contacts according to the diagnostic method using a pie chart. We tested whether or not there was any difference between the diagnostic methods on the number of contact points using the $\mathrm{T}$ test for independent samples and without assuming equal variances (to which we reported the means \pm standard deviations of the subgroups, the difference of the means [IC95\%] and the P values of the T tests) and the Mann-Whitney test (to which we reported the medians of the 2 subgroups and the $P$ value adjusted for equalities). Similarly, we used bar charts for the distribution of subjects according to the number of contact points and the method of diagnosis on all patients and in each group. We measured the magnitude of the association using the Chi-square test ( $p$ value). We did the same for the distribution according to the parameters of protrusion, right and left laterotrusion, through histograms, using the Chi-square test ( $p$ value). We did the same for the distribution according to parameters of protrusion, right and left laterotrusion, by means of histograms, using the Chi-square test in order to evaluate the differences between the diag- 
nostic methods and expressing the results by comparative graphs.

\section{RESULTS}

The sum of contact points obtained in maximum intercuspation using the calibrated $0.4-\mathrm{mm}$ articulating paper for group 1 and group 2 was compared with the sum of contact points obtained in maximum intercuspation using the T-Scan Novus device for group 1 and group 2 (Table 1).

TABLE 1. Comparison of sums of all contact points recorded in $\mathrm{MI}$ (maximum intercuspation) in both groups using the $40 \mu \mathrm{m}$ articulating paper and the T-Scan Novus device

\begin{tabular}{|l|c|c|}
\hline & $\begin{array}{c}\text { MI AP of } \mathbf{4 0} \boldsymbol{\mu m} \\
\text { (total contact points) }\end{array}$ & $\begin{array}{c}\text { MI T-Scan } \\
\text { (total contact points) }\end{array}$ \\
\hline Group 1 & 129 & 139 \\
\hline Group 2 & 128 & 111 \\
\hline Total & 257 & 250 \\
\hline
\end{tabular}

In the analysis of premature contacts in maximum intercuspation, we compared the sum of premature contacts in maximum intercuspation for both groups using 0.4-mm articulating paper and afterwards using the T-Scan Novus (Table 2).

TABLE 2. Comparison of the amounts of premature contacts recorded in both groups using $40-\mu \mathrm{m}$ articulating paper and the T-Scan Novus device

\begin{tabular}{|l|c|c|}
\hline & AP of $\mathbf{4 0} \boldsymbol{\mu m}$ & T-Scan Novus device \\
\hline Group 1 & 11 & 9 \\
\hline Group 2 & 4 & 8 \\
\hline Total & 15 & 17 \\
\hline
\end{tabular}

Regarding the distribution of contact points, balance was found between the 2 methods studied, as well as between both groups. Patients in group 2 presented a greater number of contact points when the articulating paper method was used However, the difference between the number of contact points between the 2 types of investigations (articulating paper and the T-Scan device) was not statistically significant when it was evaluated on the whole group of patients (T-test $p=0.73$, average of articulating paper group $=25.7$, average of T-Scan group = 25) (Fig. 1).

Also, the difference between the number of contact points between the 2 types of investigations (AP and T-Scan) was not statistically significant when each group was studied: group 1 - T-test $\mathrm{p}=0.54$, average of AP group $=25.8$, average $\mathrm{T}$-Scan group $=$ 27.8, group 2 - T-test $\mathrm{p}=0.26$, average of AP group $=$ 25.6, average of T-Scan group $=22.2$ ) (Fig.2 and Fig. 3 ). However, in group 2, there were a larger number of contact points diagnosed by the AP method.

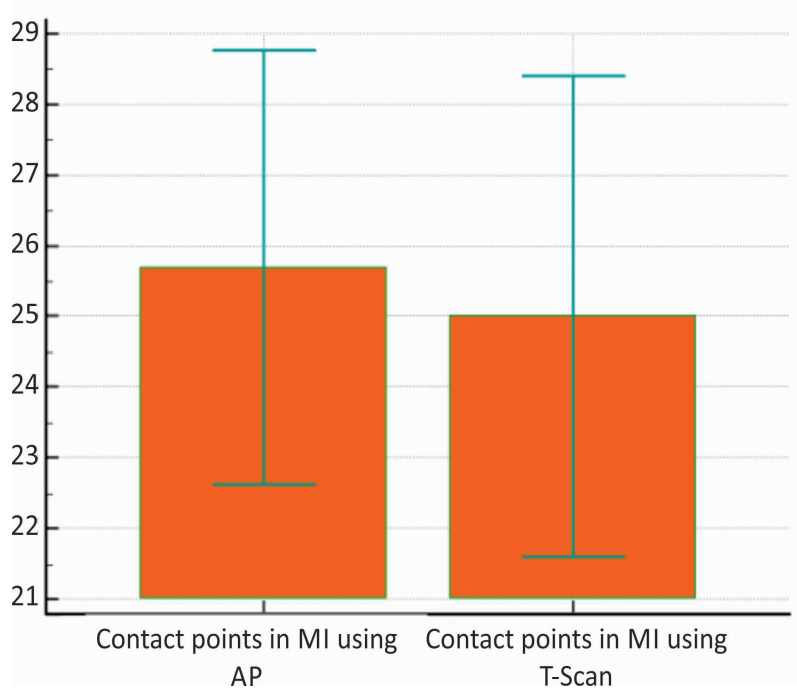

FIGURE 1. The relationship between the number of contact points in $\mathrm{MI}$ and the investigation method (AP and T-Scan Novus device)

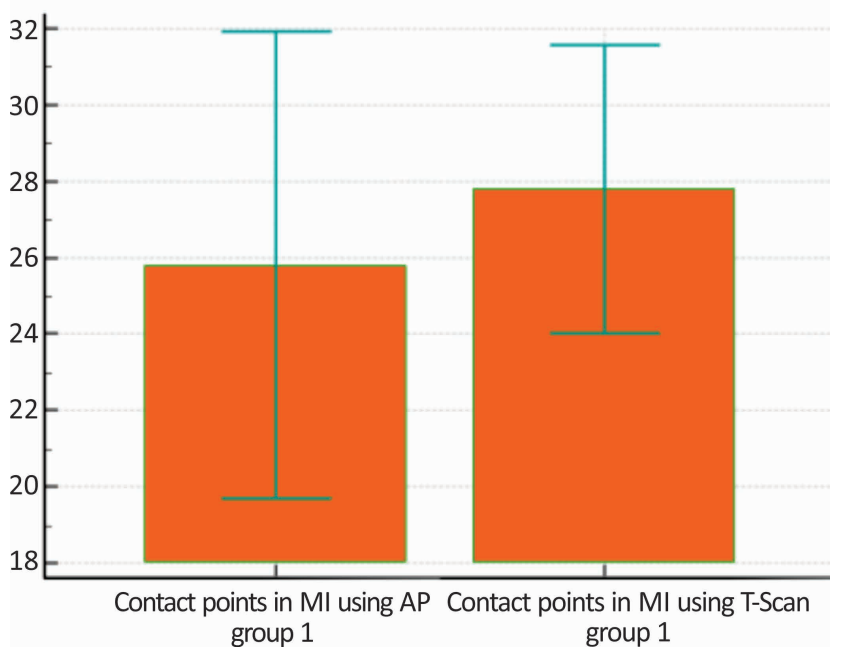

FIGURE 2. The relationship between the number of contact points and the method of investigation for group 1

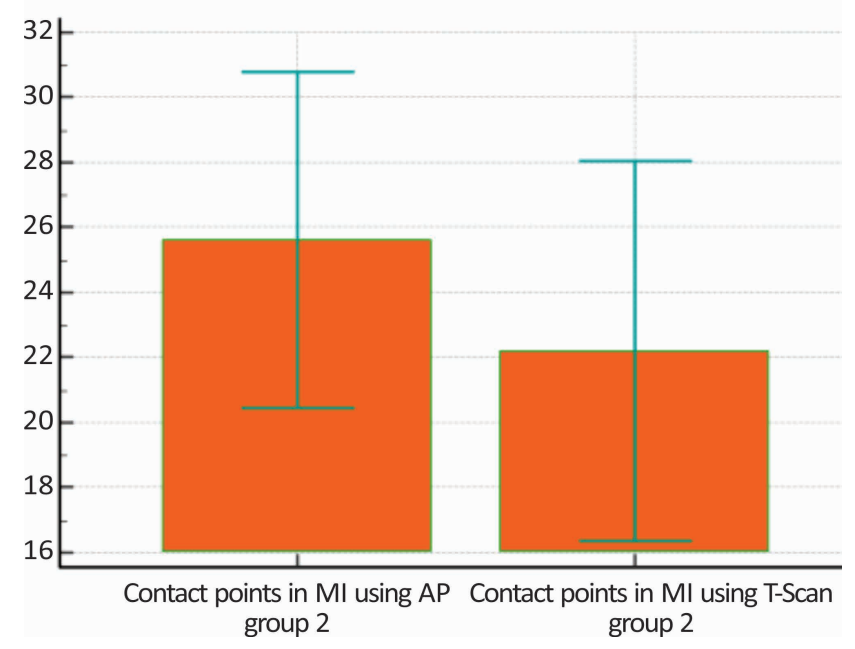

FIGURE 3. The relationship between the number of contact points and the method of investigation for group 2 
Regarding the distribution of the number of premature contacts, this was also found to be balanced in both methods studied, as well as in both groups. Patients in group 2 had more premature contact points diagnosed by the T-Scan method (Fig.4). There was no statistical significance between the 2 diagnostic methods regarding premature contacts (Chi-square $\mathrm{p}=0.27$ ).

We compared the sum of the pairs of teeth that partake in the protrusion, of the active/passive interferences, and of the active/passive premature propulsive contact points obtained with the articulated paper calibrated at $40 \mu \mathrm{m}$ for group 1 and group 2, with the same sum of contacts recorded with the T-Scan Novus device for group 1 and group 2 (Table 3).

The distribution of the parameters determined by each method within the two groups is shown in Fig. 4 and Fig. 5.

There was no statistical significance between the 2 diagnostic methods regarding protrusion (Chisquare $p=0.89$ for tooth pairs, 0.65 for API, 0.69 for APCP).
TABLE 3. Comparison of the total sums of tooth pairs involved in the protrusion movement, active and passive interferences in protrusion, active and passive premature contacts in protrusion of both groups when using the 40$\mu \mathrm{m}$ articulating paper and when using the T-Scan Novus device

\begin{tabular}{|l|c|c|}
\hline Protrusion Group 1 and Group 2 & AP & T-Scan \\
\hline Tooth Pairs & 32 & 28 \\
\hline Active Propulsive Interferences (API) & 3 & 6 \\
\hline Passive Propulsive Interferences (PPI) & 1 & 1 \\
\hline Active Propulsive Contacts Points (APCP) & 5 & 3 \\
\hline Passive Propulsive Contact Points (PPCP) & 0 & 0 \\
\hline
\end{tabular}

We compared the sum of the pairs of teeth that performed right laterotrusion, of the active/passive interferences, and of the active/passive premature contacts in right laterotrusion with the articulating paper calibrated at $40 \mu \mathrm{m}$ for group 1 and group 2, with the same sum of contacts recorded with T-Scan Novus device for group 1 and group 2 (Table 4).

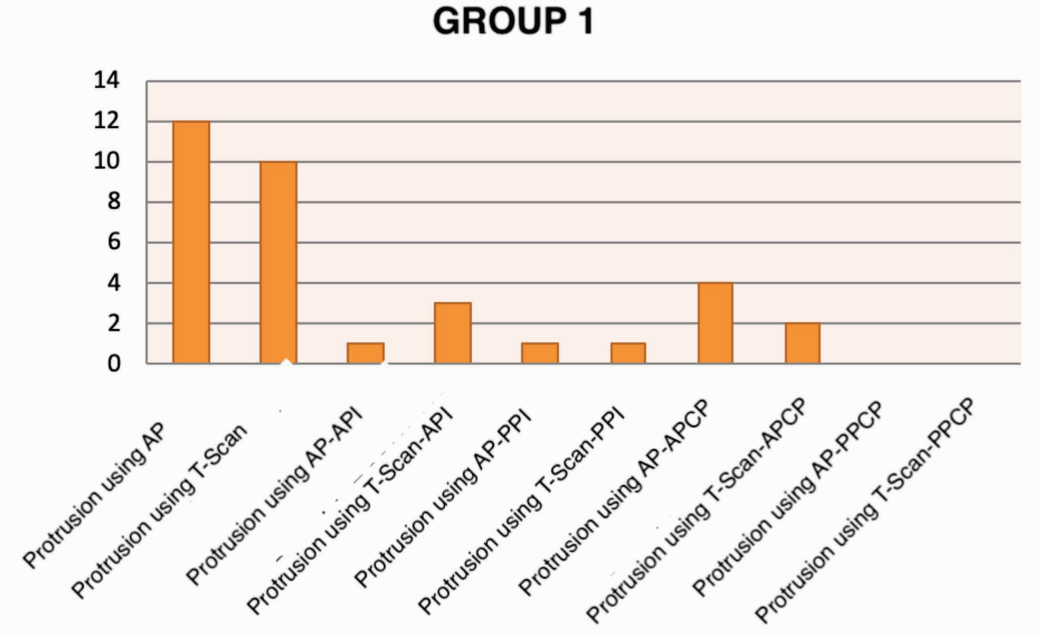

GROUP 2

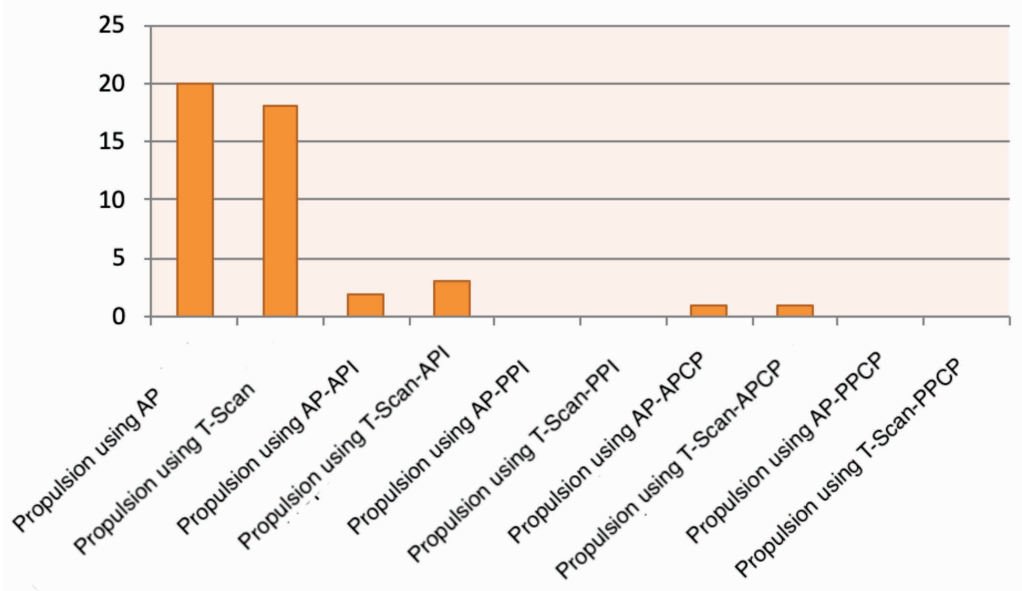

FIGURE 4. Distribution of protrusion parameters by the 2 methods in group 1 . 
TABLE 4. Comparison of the total sums of tooth pairs involved in right laterotrusion, active and passive interferences in right laterotrusion, passive and active premature contacts in right laterotrusion of both groups when using $40 \mu \mathrm{m}$ articulating paper and when using the T-Scan Novus device

\begin{tabular}{|l|c|c|}
\hline Right laterotrusion Group 1 and Group 2 & AP & T-Scan \\
\hline Tooth pairs & 27 & 30 \\
\hline Active laterotrusive interferences (ALI) & 5 & 4 \\
\hline Passive laterotrusive interferences (PLI) & 0 & 3 \\
\hline Active laterotrusive premature contacts (ALPC) & 1 & 1 \\
\hline Passive laterotrusive premature contacts (PLPC) & 0 & 0 \\
\hline
\end{tabular}

The distribution of the parameters determined each method within the two groups is shown in Fig. 6 and Fig. 7.

There was no statistical significance between the 2 diagnostic methods for right laterotrusion (Chisquare $\mathrm{p}=0.87$ for tooth pairs, 0.56 for ALI, 0.78 for IPL, 0.69 for ALPC).

We compared the sum of the pairs of teeth that performed left laterotrusion, of the active/passive interferences, and of the active/passive premature contacts in left laterotrusion with the articulating paper calibrated at $40 \mu \mathrm{m}$ for group 1 and group 2, with the same sum of contacts recorded with the T-Scan Novus device for group 1 and group 2 (Table 5).
TABLE 5. Comparison of the total sums of tooth pairs involved in left laterotrusion, active and passive interferences in left laterotrusion, passive and active premature contacts in left laterotrusion of both groups when using the $40-\mu \mathrm{m}$ articulating paper and when using the T-Scan Novus device

\begin{tabular}{|l|c|c|}
\hline Left laterotrusion Group 1 and Group 2 & AP & T-Scan \\
\hline Tooth pairs & 27 & 28 \\
\hline Active laterotrusive interferences (ALI) & 6 & 4 \\
\hline Passive laterotrusive interferences (PLI) & 0 & 3 \\
\hline Active laterotrusive premature contacts (ALPC) & 0 & 0 \\
\hline Passive laterotrusive premature contacts (PLPC) & 0 & 0 \\
\hline
\end{tabular}

The distribution of the parameters determined by each method within the two groups is shown in Fig. 8 and Fig. 9.

There was no statistical significance between the 2 diagnostic methods regarding left laterotrusion (Chi-square $\mathrm{p}=0.36$ for tooth pairs, 0.32 for PLI).

\section{DISCUSSIONS}

According to Trpevska et al. (12), the advantages of the T-Scan system are its rapidity and accuracy in identifying the distribution of the tooth contacts, being a clinical diagnostic screening device for occlusion and for improving the occlusion after ortho-

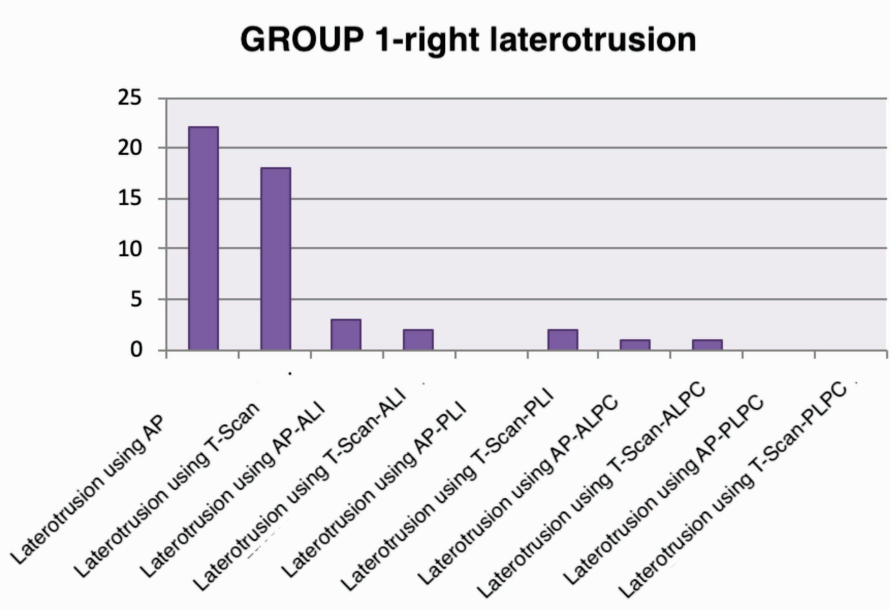

GROUP 2-right laterotrusion
FIGURE 6. Distribution of right laterotrusion parameters using the 2 methods in group 1

FIGURE 7. Distribution of right laterotrusion parameters using the 2 methods in group 2 


\section{GROUP 1-left laterotrusion}

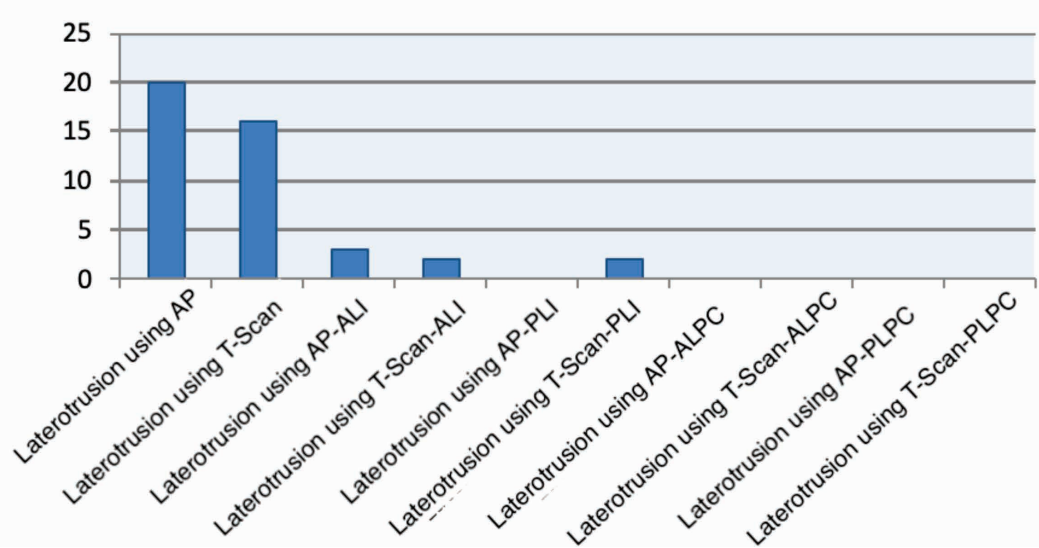

FIGURE 8. Distribution of left laterotrusion parameters using the 2 methods in group 1

\section{GROUP 2-left laterotrusion}

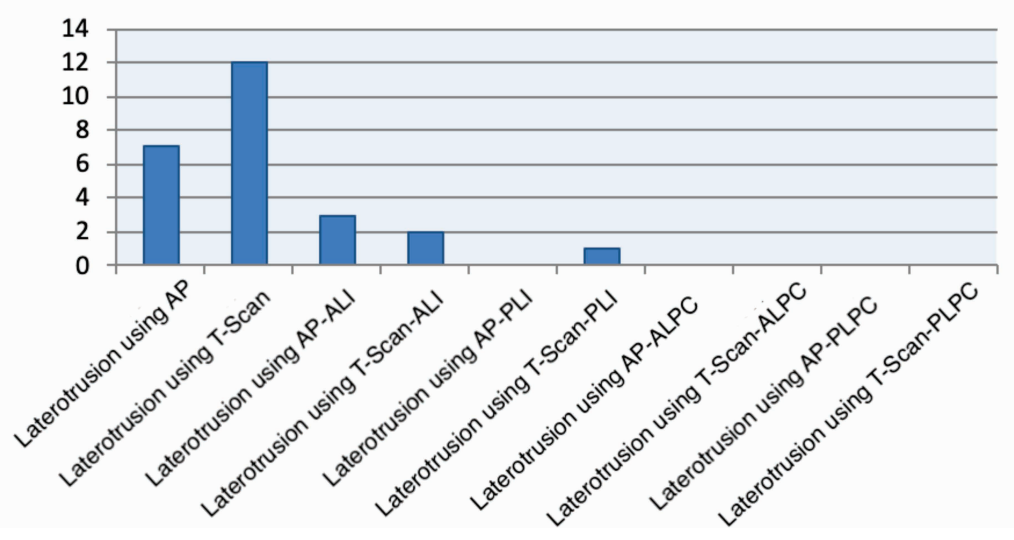

FIGURE 9. Distribution of left laterotrusion parameters using the 2 methods in group 2 dontic or prosthetic treatment. Nevertheless, additional clinical studies are required to extrapolate the clinical indications for this system.

According to Qadeer et al. (13), although computerized analysis of dental occlusion has many advantages in terms of real-time occlusal dynamics on a given interface, chronology of interarch contacts, occlusal force intensity, and occlusal balance expressed as a percentage between the right and left side, however, the most common disadvantages should also be mentioned, such as the operator-dependent factor, the standard shape of the intraoral sensor that is not suitable for all arch shapes, and the intraoral positioning of the sensor which can create discomfort and confusion for the patient during its movements. Thus, it is recommended to make repeated recordings to ensure the reproducibility of the procedure.

Another study conducted by Majithia et al. (14) concluded that the detection of premature contacts in maximum intercuspation is not sufficient with the articulating paper when followed by occlusal adjustments. Thus, computerized occlusal analysis provides complete information due to its ability to indicate with great precision the area of over occlusion. It is not very technically sensitive because the thickness of the sensor is calibrated at 100-micrometers, while the thickness of the articulating paper varies between manufacturers. Another variable of the articulating paper is the high risk of misinterpretation due to the rapid impregnation of articulating paper with saliva, while the T-Scan sensor is synthetic and the accuracy of recording is maintained even in the presence of saliva.

Considering that the determination of occlusal forces has an essential role in the diagnosis of dental occlusion, the literature review started by Trpevska et al. (12) summarizes the ways to determine the occlusal forces using the T-Scan system. Out of the 20 randomized controlled trials (RCTs) found, only 10 were included in the study. During maximum intercuspation, occlusal force and major occlusal factors are recorded with the T-Scan system, counteracting the limitations of articulating paper.

When the dental occlusion is analyzed with the help of articulating paper, a degree of subjectivity intervenes in the interpretation of the signs imprinted in the area with the strongest occlusal con- 
tacts. A study by Sutter et al. (15) regarding the accuracy of the interpretation of the signs imprinted by the articulating paper shows that the diagnosis of the most intense occlusal forces with the help of the articulating paper is limited. The average ability to correctly detect the area of the most intense occlusal force with articulating paper was $13.13 \%$, thus suggesting that dentists do not have the ability to correctly detect the area of the most intense occlusal contact by using the articulating paper alone. The results of this study are different from the results of our study.

However, the study led by Majithia et al. (14) shows that there are no statistically significant differences between premature contacts detected with 50-micrometers articulating paper and those detected with the T-Scan device on a study lot of 30 patients divided into two groups: the first group of subjects with complete permanent dentition of 32 teeth and the second group of subjects with maxillofacial trauma with complete permanent dentitions of 32 teeth. The results of this study are similar to the results of the present study, suggesting that there is a strong correlation between the articulating paper marking and the T-Scan recording; this fact becomes useful in implantology where the occlusion on the implant-supported dental crowns can be established with the help of computerized analysis. A final advantage of the T-Scan system is the ability to detect with maximum precision the greatest force at the level of a pair of antagonistic teeth.

This advantage is also mentioned in the conclusions of the study led by Qadeer et al. (13), stating that computerized occlusal analysis can provide additional valuable information regarding the intensity of the force and its precise location.

\section{CONCLUSIONS}

The analysis of maximum intercuspation, protrusion and laterotrusion had comparable results when using the two examination methods: the analog method by means of the calibrated articulating paper, and the digital method by means of the T-Scan Novus v10 device. The differences between them are, however, not statistically significant.

For the diagnosis and treatment through occlusal equilibration it is necessary to correlate the analog method of occlusal analysis with the digital one. Nevertheless, for a complete and comprehensive occlusal analysis we would recommend the synergistic use of both methods.

\section{Acknowledgement}

All authors have equal contribution to this study.

Conflict of interest: none declared Financial support: none declared

\section{REFERENCES}

1. Rekow ED. Digital dentistry: The new state of the art - Is it disruptive or destructive? Dent Mater. 2020 Jan;36(1):9-24.

2. Maness WL, Benjamin M, Podoloff R, Bobick A, Golden RF. Computerized occlusal analysis: a new technology. Quintessence Int. 1987 Apr;18(4):287-92.

3. Reza Moini M, Neff PA. Reproducibility of occlusal contacts utilizing a computerized instrument. Quintessence Int. 1991 May;22(5):357-60.

4. Kerstein RB, Radke J: Clinician accuracy when subjectively interpreting articulating paper markings. Cranio. 2014;32:13-23.

5. Schelb E, Kaiser DA, BrukI CE. Thickness and marking characteristics of occlusal registration strips. J Prosthet Dent. 1985 Jul;54(1):122-6.

6. Sheridan RA, Decker AM, Plonka AB, Wang HL. The Role of Occlusion in Implant Therapy: A Comprehensive Updated Review. Implant Dent. 2016 Dec;25(6):829-838.

7. Qadeer S, Yang L, Sarinnaphakorn L, et al. Comparison of closure occlusal force parameters in post-orthodontic and non-orthodontic subjects using T-scan R_ III DMD occlusal analysis. Cranio. 2016;34:395-401.

8. Qadeer S, Abbas AA, Sarinnaphakorn L, et al. Comparison of excursive occlusal force parameters in post-orthodontic and non-orthodontic subjects using T-ScanR_III. Cranio. 2018;36:11-18.

9. Cerna M, Ferreira R, Zaror C, et al. Validity and reliability of the T-Scan R_ III for measuring force under laboratory conditions. J Oral Rehabil. 2015;42:544-551.
10. Qadeer S, Kerstein R, Kim RJ, Huh JB, Shin SW. Relationship between articulation paper mark size and percentage of force measured with 4(1):7-12.

11. Carey JP, Craig M, Kerstein RB, Radke J. Determining a relationship between applied occlusal load and articulating paper mark area. Open

12. Trpevska V, Kovacevska G, Benedeti A, Jordanov B. T-scan III system diagnostic tool for digital occlusal analysis in orthodontics - a modern approach. Pril (Makedon Akad Nauk Umet Odd Med Nauki). 2014; 35(2):155-60. articulation paper mark size and percentage of force measured with computerized occlusal analysis. J Adv Prosthodont. 2012 Feb; 4(1):7-12.

14. Majithia IP, Arora V, Anil Kumar S, Saxena V, Mittal M. Comparison of articulating paper markings and T Scan III recordings to evaluate occlusal force in normal and rehabilitated maxillofacial trauma patients. Med J Armed Forces India. 2015 Dec;71(Suppl 2):S382-8.

15. Sutter BA. A digital poll of dentists testing the accuracy of paper mark subjective interpretation. Cranio. 2018 Nov;36(6):396-403. computerized occlusal analysis. J Adv Prosthodont. 2012 Feb; Dent J. 2007;1:1-7.

13. Qadeer S, Kerstein R, Kim RJ, Huh JB, Shin SW. Relationship between 